\title{
APECTS OF THE EASTERN QUESTION FOUND IN SWEDISH DIPLOMATIC A reports (1813)
}

\section{Veniamin Ciobanu}

“A. D. Xenopol” Institute of History, e-mail: veniamin@xenopol.iasi.astral.ro

\begin{abstract}
:
The documents selected by the editor from the National Archives of Sweden offer information reflecting the preoccupation of the Swedish diplomacy to be well informed with regard to the Eastern Question developments. In 1813 such information was necessary in order to guide the Swedish government's policy in reaching its essential goal, the integration of Norway in the Swedish political system. In order to have a broader perspective on the international events and circumstances of the period marking the end of the Napoleonean Empire is necessary that these documents shall be corroborated with other documentary sources, such as, for instance, the ones included in the seventh volume of the foreign policy documents collection entitled "Europe and the Porte. New Documents on the Eastern Question".
\end{abstract}

\section{Résumé:}

Les documents sélectés par l'éditeur des Archives Nationales de la Suède offrent des informations qui reflètent la préoccupation de la diplomatie suédoise d'être bien informée sur le cours du développement de la question orientale. Pendant l'année 1813 ces informations ont été nécessaires pour l'orientation de la politique du Cabinet de Stockholm en vue d'accomplir son but essentiel d'alors, c'est-à-dire l'intégration de la Norvège dans le système politique suédois. Mais pour avoir une perspective plus large sur les événements et les circonstances politiques internationales de la période qui a marqué la fin de l'Empire Napoléonien il est nécessaire que ces documents soient corroborés avec d'autre sources documentaires, comme par exemple celles incorporées dans le tome VII de la Collection de documents extérieurs intitulée "Europe and the Porte. New Documents on the Eastern Question".

\section{Rezumat:}

Documentele selectate de editor din Arhivele Naționale ale Suediei oferă informații care reflectă preocupările diplomației suedeze de a fi bine informată cu privire la cursul evoluțiilor "chestiunii orientale". In anul 1813 aceste informații erau necesare pentru orientarea politicii cabinetului de la Stockholm pentru a-şi realiza scopul său esențial, integrarea Norvegiei în sistemul politic suedez. Pentru a avea 
o perspectivă mai largă asupra evenimentelor şi a circumstanțelor politice internaționale ale perioadei care a marcat sfârşitul Imperiului Napoleonian, este necesar ca aceste documente să fie coroborate cu alte surse documentare, precum, de exemplu, cele incluse în volumul al VII-lea al Colecției de documente externe intitulată "Europe and the Porte. New Documents on the Eastern Question".

Keywords: The Eastern Question, Swedish diplomatic reports, Sweden, Turkey

The documents that we submit to the attention of the international relations history researchers, in general, and to those who research the Eastern question, in particular, were produced during a chronological stage set apart in the history of Sweden by its amplest involvement to that time in the deployment of the last stage of the European political system crises, and also of the Oriental issue, caused by the Napoleonic wars. In the respective context, Sweden's foreign policy was also radically reconsidered and it abandoned its anti-Russian orientation adhering, along with Russia and Great Britain, to the $6^{\text {th }}$ anti-French coalition, established during the months of February-July 1812. The person who influenced this orientation was one of France's famous marshal and Napoleon Bonaparte's close collaborator, namely Jean Baptiste Jules Bernadotte, prince of Pontecorvo, who was officially proclaimed, by Sweden's riksdag, as inheritor of its Crown, on March 16, 1811, under the name of Karl Johan ${ }^{1}$.

His being chosen and proclaimed as inheritor prince occurred against the background of the deterioration of the France and Russia relationship, and also that of the Swedish hopes that the new inheritor prince will manage, with the support of Napoleon Bonaparte, to bring Finland back within the frontiers of the Kingdom of Sweden, as Finland had been annexed by Russia in the year $1807^{2}$. However, the leading Swedish circles had in view another variant, that of compensating for the loss of Finland, in case it would no longer be possible to recuperate it from Russia, with the annexation of Norway, which was part of the Kingdom of Denmark. Nevertheless, given that the idea of the recuperation of Finland, with the help of France, had to be completely abandoned, because of the occupation of the Swedish Pomerania by the French troops in the summer of 1811, the annexation of Norway became the priority objective for the foreign policy promoted by Karl Johan. Moreover, as this priority could only have been achieved with the support of Russia and Great Britain, the

\footnotetext{
1 "Introduction“, in Europe and the Porte. New Documents on the Eastern Question, Volume VII: Swedish Dilomatic Reports 1811-1814, eds. Veniamin Ciobanu, Leonidas Rados and Alexandru Istrate (Iaşi: Editura Junimea, 2009), 7.
}

2 Ibid., $2,7$. 
approach of Russia and the adhesion to the anti-French coalition became an essential condition. Therefore, from one adversary, Sweden turned into one ally of Russia against France ${ }^{3}$.

The French attack, which became imminent in the summer of 1812, determined the regent prince of Sweden to get even more involved in the efforts to convince the Russian tsar, Alexander I, not only to conclude the peace with the Ottoman Empire, with which it had been at war since 1806, but even a military alliance with it, so as to be more successfully capable to face the danger represented by the French aggression. And, in order to hasten the materialization of that project, Karl Johan sent a few diplomatic missions to Istanbul to determine the Porte as well to hasten the conclusion of the peace, under the pretext that Napoleon's "universalistic" plans were similarly dangerous for the security and territorial integrity of the Sultan's Empire. Among one of those to whom this mission was entrusted was Karl Johan Tavast, former governor of the port-town Göteborg, remarked for his intransigent anti-Napoleonic attitude ${ }^{4}$.

This time, the main mission of the new envoy consisted in determining the Porte to adhere to the anti-French coalition. He arrived in Istanbul on June 23, 1812, after the conclusion of the peace between Russia and the Porte, on May 28 the same year. Just like his predecessors, he was not able to accomplish his mission, so he had to leave Istanbul at the beginning of the month of February $1813^{5}$.

Thus, the attempts of the Swedish diplomacy failed, both because of the opposition of the Porte, which wanted to maintain its position of extremely strict neutrality, and because of the military incapacity thereof, as well as of the severe economic effects due to the long war with Russia and to Russia's abandonment of the project. All this because it gave up the idea of a Russian diversion against the French armies operating in Dalmatia, due to the Porte's opposition towards the Russian troops crossing the territory of its South-Danube provinces owing to the perpetuation of some Russian-Turkish litigations, unsettled by the peace treaty of May 28, 1812. Add to this the fact that Great Britain did not like the idea of the Porte's involvement in the anti-French war, because of the complications to the British interests in Eastern Europe. Consequently, in the month of February, 1813, the Swedish diplomacy abandoned, as well, the project of involving the Porte in the anti-French coalition'.

\footnotetext{
${ }^{3}$ Ibid., 8 and the following.

4 Ibid., 16-19.

5 Ibid., 19-20.

${ }^{6}$ Ibid., 21-22.
} 
Even if it was no longer taken into consideration from this perspective, however, it remained just as before an important field of action for the Swedish diplomacy. That is because the position of Sweden in the anti-French coalition seemed vulnerable enough in the year 1813, as it was possible for it to lose Russia's support in the Norway annexation, which was, as we have mentioned, the essential objective of its adhesion to the anti-French war. This is why the Swedish diplomacy pursued, by its representative to Istanbul, the chargé d'affaires Nils Gustaf Palin, as Sweden's goal was to hold the best relations with the Porte, whereas it continued to consider it as capable of offering, in case of need, diplomatic support in order to bring about the objective mentioned above. This accounts for the attention with which Nils Gustaf Palin watched both the evolution of the internal political and economical situation of the Ottoman Empire and the deployment of the Porte's relations with Russia, and also with other states, like Great Britain, recording, at the same time, any other event that could have interested the leaders of Sweden's foreign policy . This explains the information included in the reports addressed to his superiors from Stockholm, concerning some aspects of the Eastern question. Information that, corroborated with other sources, like, for instance, those included in the $7^{\text {th }}$ volume of the collection Europe and the Porte. New Documents on the Eastern Question can contribute to the expansion of the research horizon for the history of the Eastern question. Information whose value, I hope will be, from this viewpoint, seized by those who will use it.

Therefore, this is a very brief review of the diplomatic reports of the occurrence circumstances, which we brought to the readers' attention, in order to facilitate their understanding of these reports' contents.

The documents, selected from Sveriges Riksarivet of Stockholm, which represent both original pieces and copies, having, however, the same value of work tools, in the case of those from the latter category, have not been paginated by the Swedish archivists. When transcribing them, we kept, as much as possible, the punctuation, the orthography, the syntax and the language of the originals. Where such was the case, we completed certain words and expressions by putting the missing parts between square brackets; the expression "sic!" was used to mark expressions or words that could not be replaced.

In the end, as you shall notice, each document is accompanied by a regest and by explicative notes. 
Constantinople, le 25 Janvier 1813

Nils Gustav Palin, chargé d'affaires de la Suède auprès de la Porte, à [Lars von Engestróm, ministre d'Etat et ministre des Affaires Etrangères de la Suède]

Le refus de la Porte de se conformer à certaines normes diplomatiques sollicitées par lui; la motivation de l'attitude respective; son opinion, selon laquelle le refus en discussion pourrait être expliqué par la crise financière à laquelle la Porte était confrontée alors; ses tentatives de déjouer les intrigues françaises dirigées contre la Suède, en vue de prévenir l'adhésion de la Porte à la coalition antifrançaise, l'objectif d'alors de la diplomatie suédoise

\section{Constantinople le 25 Janv[ier] 1813 Apostille de M[onsieur] de Palin}

Dans la même occasion que le Général de Tavast ${ }^{7}$ avait demandé Ses audiences de congé, j'ai insinué, à Son inscu, par une lettre ostensible adressée à Seraphino8, la convenance de le décorer du grand cordon de l'ordre du Croissant et de donner la petite croix à M[onsieu]r le Comte Rosen ${ }^{9}$. Le désir si naturel de voir l'envoyé du Roi ${ }^{10}$, traité de la manière la plus honorable, m'avoit fait appuyer cette proposition de tous les motifs possibles ; mais il a été répondu que cet ordre était entièrement aboli. Il n'a pas été donné en effet, depuis la mort du Sultan Sélim ${ }^{11}$ que le goût pour les usages Chrétiens avait rendu odieux à Sa nation; et en allant aux informations, j'ai appris que les Russes avaient stipulé des décorations dans les pourparlers (sic !) de la paix ${ }^{12}$, mais que le Grand Segneur avait refusées ensuite par la même raison de l'abolition.

Par une nouvelle lettre à Seraphino j'ai donc rappelé délicatement le présent d'usage tel qu'il pouvait supplier à l'ordre, en faisant honneur aux deux Cours ${ }^{13}$. Alors le Drogueman de la Porte ${ }^{14}$ a fait entendre à celui de la Mission, que la Porte se privoit du plaisir de présenter à M[onsieu]r de Tavast une marque de Sa satisfaction, pour ne pas donner lieu de l'attribuer

\footnotetext{
7 Johan Henrik Tavast; Swedish general; former governor of the harbor of Göteborg ; sent with special missions to Istanbul.

8 Paul Serafino; first interpreter of the Diplomatic Mission of Sweden in Istanbul.

9 Swedish emissary to the Porte.

${ }^{10}$ Charles XIII; King of Sweden (1808-1818).

11 Selim III (1789-1807).

12 The Russian-Turkish Peace concluded in Bucharest on May 28, 1812 ended the war started in 1806.

13 Sweden and the Porte.

14 Iacob Arghiropol; grand drogueman (interpreter) of the Porte (1812-1815). 
à l'effet d'adhésion à la proposition de la cour de Suéde ${ }^{15}$. J'ai aussitôt relevé, dans une troisième lettre tout ce qu'il y avait de choquant dans l'idée de manquer d'égards à la Suéde, et de propos délibéré, pour ne pas faire soupçonner qu'on s'allia plus intimement avec elle et la Russie ; pour piquer la fierté Ottomanne, j'ai même ajouté, que la crainte de la France pouvoit moins que jamais arrêter le Grand Seigneur. Seraphino a fait encore usage de cette lettre, mais tout ce qu'il a pu dire a été inutile, et il est revenu de la Porte convaincu que c'étoit un parti arrêté entre tout le Ministère, de ne pas donner le présent, Le Reis Effendi ${ }^{16}$ seul ne pouvant pas le prendre sur lui.

Vivement peigné de cet incident, je ne sçais à quoi l'attribuer ; aux intrigues du parti Français, à la manière de voir de la Porte, qui n'est pas accoutumée à recevoir des organes diplomatiques sans titre; à la haine fanatique contre la Russie, dont le Grand Seigneur lui même, n'est pas exempt; à la lésine, enfin, aussi extrême que la pénurie incroyable des fiances, et qui avoit fait supprimer les présens de congé pour Messieurs $\mathrm{d}^{\prime}$ Ohsson ${ }^{17}$ et König ${ }^{18}$. Toutes ces circonstances ensemble ont pu déterminer l'orgueilleuse indifférence et la grossièreté du Ministère ${ }^{19}$ : ces qualités garantissent dumoins (sic !) la sincérité des protestations renouvellées (sic !) de sensibilité à la démarche de la Suéde et l'estime pour le Général qui a été très agréable au Ministère.

Ayant reçu peu après les ordres de V[otre] E[xcellence] du 17 Nov[embre], je n'en ai fait part à la Porte qu'en accompagnant cette communication d'un billet adressé à Seraphino que par un autre je l'ai instruit de montrer, mais sous le secret, et comme n'y étant nullement autorisé. Je crois devoir soumettre à Votre] E[xcellence] le premier billet tout entier de la teneur suivant.

“J'ai appris par M[onsieu]r le Général de Tavast, qui m'a transmis les lettres arrivées par le Courier de Vienne, qui Vous avez déjà communiqué à la Porte, le contenu de celles de Monsieur Hégardt ${ }^{20}$; et qu'on a désiré la Copie que je suis faché de ne pas pouvoir donner, puisqu'avec des notions incomplètes sur les événemens de la guerre ${ }^{21}$, cette lettre renferme des

\footnotetext{
15 Of determining the Porte to accede to the anti-French coallition.

16 Mehmed Efendi (Kücük - Arif; reis efendi of the Porte (VI/VII. 1811-22. I. 1814).

17 Ignatius Mouradgea d'Ohsson; former envoy plenipotentiary of Sweden at the Porte (22.12.1796-19.07.1799).

18 Karl Gustaf König; former chargé d'affaires of Sweden at the Porte (10.07.1799-15.05.1805).

19 High (Sublime) Porte.

20 Christian Bernhard Hegardt ; chargé d'affaires of Sweden in Vienna (19. VIII. 1812-15. V. 1805).

21 The war between Napoléon Bonaparte's France and the six anti-French coallition created in February - July 1812 by Great Britain, Russia and Sweden.
} 
conjectures particulières à M[onsieu]r Hegardt desquelles il ne m'a pas dit qu'il veut être responsable. Je Vous avouerai même, que je suis embarrassé après ce qui vient d'arriver, de faire part d'une dépêche de la Cour, qui jusqu'à nouvel ordre je devrois peut être considérer comme faite pour moi seul, et pour me tenir au fait de l'état des Affaires; il devient superflu naturellement, que la Suéde éclaire la Porte sur Sa véritable position, si ces marques d'amitié lui sont indifférentes et si elle croit devoir y repondre par des malhonnetetés affectées, pour complaire à une puissance qui emplois tour à tour l'imposture et la violence pour tout tromper et tout soumettre ; qui fait répandre en Suéde, que le Grand Seigneur a fait une visite à l'Ambassadeur de France 22 , tandis qu'elle a soin de faire venir ici par estafette la nouvelle de troubles réels en Suéde. Vous verrez ce manége dans la copie de la Dépeche, ci jointe, que Vous pouvez bien montrer à Monsieur Argiropolo, mais sans absolument en donner copie. La communication ainsi faite est tout ce que je puis prendre sur moi, ou laquelle malgré les circonstances, je n'ose pas soustraire. C'est l'amitié de la Suéde pour cet Empire ${ }^{23}$, fondé sur des rapports immuables qui depuis plus d'un siècle ont fait courir aux deux Empires ${ }^{24}$ les même chances et qui en tous tems rend naturelles des marques d'amitié. Le Ministère Ottoman sait quels sont ces rapports; quand tout a été changé dans les affaires générales, la Suéde n'a pas cru que les deux Puissances devoient s'écarter d'un systême commun, de défense, contre un ennemi nouveaux, à la vérité, mais plus redoutable".

Cet aigre doux (sic!) m'a paru convenable dans la circonstance pour remettre la Porte au véritable état de la question et pour ressentir sa conduite envers M[onsieu]r de Tavast.

J'ai refusé copie de la lettre de M[onsieu]r Hegärdt (sic !), parcequ'il m’a paru que le Drogueman de la Porte, attaché au Parti Français, vouloit profiter de quelques détails qu'elle renfermoit sur des renforts qui pourront encore rendre Napoléon redoutable et encourager la Porte à s'attacher encore plus à Son prétendu vengeur. Je préviens M[onsieu]r de Hégardt que ses lettres étant proprement faites pour le Ministère Ottoman et devant contribuer à la direction de la politique de cet Empire, il conviendra de distribuer les notions qu'il me donne, de manière à séparer celles qui seront propre à communiquer à la Porte dans cette vue d'importance majeure.

$\mathrm{V}$ [otre] E[xcellence] a déjà vu par mon billet à Seraphino que la visite du Grand Seigneur à l'Ambassadeur de France, n'a pas eu lieu. On ne s'est

\footnotetext{
22 Antoine François Andréossy; ambassador extraordinary of France at the Porte (25.07.181214.11.1814).

23 The Ottoman Empire.

24 Sweden and the Ottoman Empire.
} 
pas douté ici de la possibilité d'une telle nouvelle qui coincide avec celle qu'on fait sur les lieux de l'ordre que le Grand Seigneur aurait donné de faire baisser le pavillon que l'Ambassadeur faisait flotter sur sa maison de campagne, d'aprés son usage de Consul, que la mission de France seule a observé aprés la révolte. La Porte a été discrète sur la fausse nouvelle de trouble en Suéde.

Sveriges Riksarkivet, Kabinettet/UD, Huvudarkivet, E2D, 226, 18111813, Konstantinopel; copy in French language.

\section{2}

Constantinople, le 9 Mars 1813 Nils Gustaf Palin, à [Lars von Engestróm]

Stade des négociations russo-turques; mécontentements des Serbes concernant les stipulations en ce qui les concernait du traité de paix russo-turque, du 28 mai 1812, de Bucarest; désir de la Porte que la Russie et la France s'épuisent réciproquement à cause de la guerre déclenchée par la France, le 24 Juin 1812.

Constantinople le 28 Mars 1812 Apostille de M[onseu]r Palin

$\mathrm{M}$ [onsieu]r Italinsky25, tout en continuant de se plaindre du Ministère ${ }^{26}$ et de l'inutilité des représentations qui ne sont qu'en paroles, m'a dit cependant qu'il s'est beaucoup adouci dans la négociation qui avance vers une conclusion favorable. Il s'est exprimé sur les Serviens qu'ils s'opposaient à l'exécution du Traité de Paix ${ }^{27}$ à leur égard, et que la Porte se préparait à les contraindre par la force, sans qu' on puisse préjuger ses succès, À la Porte on m'a avoué leur obstination, mais sans convenir des voies de fait, et de la conclusion de cette affaire, dont on parle dans le public.

À cette occasion le Drogueman de la Porte 28 m'a fait dire $q u^{\prime}$ on attribuoit encore à une partie de la nation Suedoise du mécontentement sur le Systême adopté par le Gouvernement. J'ai fait dire en réponse qu'on se fondoit tout au plus sur le langage de rancune contre la Russie parmi quelques particuliers, auxquels on ne peut pas supposer les mêmes lumières et la grandeur d'âme du Gouvernement qui a su oublier les torts

\footnotetext{
25 Andrei Iakovlevici Italinski; envoy extraordinary and minister plenipotentiary of Russia at the Porte (7.08.1812-10.04.1814).

26 High (Sublime) Porte.

27 The Russian-Turkish Peace Treaty signed in Bucharest on May 28, 1812.

28 Iacob Arghiropol.
} 
de cette Puissance dans un moment où elle a eu à combattre pour les interêts de tous les autres.

Le Reis Effendi 29 est entré en grandes discussion avec Seraphino ${ }^{30}$ sur le peu de probabilité de la réussite de la médiation Autrichienne, dont je lui avois communiqué les premieres notions recues quinze jours avant que $l^{\prime}$ internonce ${ }^{31}$ en a été informé par Sa Cour. Les voeux secrets de la Porte paraissent être en effet que la Russie et la France s'épuisent également par cette guerre.

Sveriges Riksarkivet, Kabinettet/UD, Huvudarkivet, E2D, 226, 18111813, Konstantinopel; copy in French language.

3

Constantinople, le 26 Mars 1813

Nils Gustaf Palin, à [Lars von Engeström]

Décision du roi de la Prusse de se joindre à la coalition antifrançaise, constituée aux mois de février-juin/juillet 1812, par l'Angleterre, la Russie et la Suède, par l'intermédiaire d'une alliance avec la Russie; la situation politique de l'Empire Ottoman et ses opinions concernant celle-ci: informations détenues par la Porte concernant les résultats de l'entrevue de Carol Johan, prince héritier de la Suède, avec le tsar Alexandre I.

Constantinople le 26 Mars 1813

Très humble Apostille de M[onsieu]r Palin

Monsieur Italinski32, qui m'a informé dela détermination, secrette encore, de S[a] M[ajesté] le Roi de Prusse ${ }^{33}$ de se joindre à la Russie avec une Armée de 100,000 hommes, et de l'aplanissement de ses discussions avec la Porte, a cependant parlé avec quelque dépit des différentes mésures que prend ce Gouvernement ${ }^{34}$ pour ramasser des troupes et de l'argent, pour remettre l'ordre dans les Provinces et reprendre une attitude imposante vis-à-vis de l'Etranger, dans des vues soient (sic !) d'attaquer ou de défence. Parmi les expédiens financieres, a été la décapitation d'un riche

\footnotetext{
${ }^{29}$ Ahmed Efendi (Kücük-Arif).

30 Paul Seraphino.

31 Ignaz Lorenz Stürmer; ad-interim diplomatic representative of Austria at the Porte (30.09.1802-10.03.1818).

32 Andrei Iakovlevici Italinski.

33 Friedrich Wilhelm III; King of Prussia (1796-1940).

34 High (Sublime) Porte. 
Particulier à Galipoli, sans autre crime connu que sa fortune supposé de 15 millions.

Le Gouverneur de Silistria nommé au commendement de Widdin a réussi de gagner les habitans et une partie dela Garnison de cette ville, de sorte qu'ils ont declaré a leur Pacha qu'ils n'entendent point se trouver impliqués dans la Rébellion contre la religion et le Souverain.

La Position du Gouvernement vis-à-vis de redoutable Pascha de Ianina, peut entrainer plusieurs désagréments, entre'autre (sic!) par les liaisons des Anglais avec ce Pascha persecuté par l'Ambassade de France. Le Pascha d'Egypte ayant en vue d'obténir le Paschalik de Damas pour son Fils ainé en récompense de es exploits militaires en Arabie, la discussion avec le Pascha semble aussi menacer d'une de ces guerres intestines devenues si communes.

Le Drogueman dela Porte ${ }^{35}$ a prévenir Seraphino ${ }^{36}$ depuis plusieurs jours dela (sic !) connaissance qu'il avait du départ de M[onsieu]r Ohgsson de Paris ${ }^{37}$. A cette occasion il a aussi dit savoir qu'il etait convenu à $\mathrm{Abo}^{38}$ entre le Prince Royal ${ }^{39}$ et l'Empereur ${ }^{40}$ que la Norvège serait cedé ala (sic !) Suède contre la Pomeranie et l'Hannovre obtiendrait le Dannemarc en se joignant a l'alliance du Nord.

Ayant reçu de M[onsieu]r de Hegard ${ }^{41}$ la Note adressé à M[onsieu]r Cabre, et la Dépêche de S[on] E[xcellence] M[onsieu]r le Baron d'Engeström en date de 22 Janvier, j'en ai fait part àla (sic !) Porte dont le Drogueman a demandé Copie de la première piece.

Sveriges Riksarkivet, Kabinettet/UD Huvudarkivet, E2D, 226, 18111813, Konstantinopel; copy in French language.

4

Constantinople, le 24 Avril 1813 Nils Gustaf Palin, au roi de la Suède

Perpétuation de la crise serbe et de la révolte du pacha de Vidin; stade des négociations russo-turques dans le domaine du commerce du blé; évolution des

\footnotetext{
35 Iacob Arghiropol.

36 Paul Seraphino.

37 Ignatius Mouradgea d'Ohsson.

38 He referes to the meeting between Carl Johan, Crown Prince of Sweden, and the Tsar Alexander I of 27 to 31.08.1812, Ingvar Andersson, A history of Sweden. Translated from the Swedish by Carolyn Hannay (Stockholm: Natur och Kukltur), 318.

39 Carl Johan.

40 Alexander I.

${ }^{41}$ Christian Bernhardt Hagardt.
} 
événements sur le théâtre de guerre entre la France et ses adversaires de la coalition antifrançaise ; situation en Egypte.

\section{Sire,}

Constantinople 24 Avril 1813

Mon dernier très humble rapport étoit du 10 de ce mois.

L'etat des choses vis-à-vis les Serviens et le Pascha de Widdin étant toujours le même à peu près, le Grand Vizir ${ }^{42}$ contenu son sejour au Camp, dont le Reis-Effendi ${ }^{43}$ a été remplacé par son Secretaire Hasni Bey, homme de talent, qui fu disgracié un moment sous le dernier Vizir ${ }^{44}$. La discussion facheuse avec la Russie nest (sic !) pas non plus entièrement terminée, par l'arrangement convenu dont j'ai eu l'honneur de rendre compte, ceux qui font le commerce de bleds à Odesse ayant protesté contre cette mesure, tant auprès de la Mission ${ }^{45}$, que du Gouvernement ${ }^{46}$. Aussi la première n'a-t-elle pas encore reçue la ratification de $l^{\prime} E$ pereur ${ }^{47}$. Il faut ésperer la solution de ces diffcultés dans une bonne recolte. En attendant les acquereurs étrangers des bleds s'en voient trouvés à moins de se soumettre à des grands sacrifices clandestins, et le commercant d'Odesse y perde, aussi indibitablement puisque le bled revient, dans ce port, au double du prix, auquel il faudra ceder içi la moitié des cargaisons, pour pouvoir faire passer l'autre partie. Cette affaire pendant,le retablissement des Consulats Russes dans les differentes echelles du Levant est retardé.

La poste Russe va être rétablie incessamant. Des courriers extraordinaires du Camp ou de Petersbourg ne sont pas arrivés dernièrement, mais l'on ne manque pas des notions sur les progrès des événemens, parmi lesquels le public croit le debarquement des troupes Suèdoises en Pomeranie déjà effectuée, depuis l'évacuation de cette province par les Francais. Un courrier Turc est arrivé de Paris dépuis plusieurs jours.

Une petite escadre qui met en rade dans ce moment, est destiné à croiser dans l'Archipel, contenir le Pascha Rebelle de Satralie, et porter des renforts de troupes et de munitions à Celui d'Egipte, dont les plans semblent s'elargir avec ses sucés contenués en Arabie, de sorte qu'on lui attribue le projet en tout temps d'une execution si difficile de conquerir aussi Yemen. Le Gouvernement vient de donner un exemple qui peut

\footnotetext{
42 Hurşid Ahmed pacha; Grand Vizier (5. IX. 1812-1. IV. 1815).

43 Ahmed Efendi (Küçük-Arif).

44 Ahmed pacha; former Grand Vizier (10. IV. 1811-5. IX. 1812).

45 Russia's Diplomatic Mission in Istanbul.

46 High (Sublime) Porte.

47 Alexandre I.
} 
dévenir salutaire, en établissement d'abord des Lazarets en toutes les institutions Européennes pour prévenir la contagion de la peste ; et en les soumettant à la direction illimité des Consuls étrangers, depuis qu'il a vu ses effort inutiles sous l'administration des Turcs. Il a expedié ici un de ses fils qu' on attend tous les jours.

Antoine Murat, ci devant interpret de la mission de Votre Majesté48 venant de mourir, la decoration de l'Ordre de Vasa, dont il a été honoré, me fera remise pour être renvoyée au chapitre des ordres.

J'ai l'honneur de ci-joindre les nouvelles publiées par la mission Russe, avec une proclamation de S[on] A[ltesse] R[oyale] le Prince Royal ${ }^{49}$, dont on a contesté l'authentecité.

Je suis avec le plus profond respect

Sire

de Votre Majesté

le très humble et très fidèle et soumis serviteur et sujet

N. G. Palin.

Sveriges Riksarkivet, Kabinettet/UD Huvudarkivet, E2D, 226, 18111813, Konstantinopel; original in French language.

5

Constantinople, le 25 Mai 1813

Nils Gustaf Palin au roi de la Suède

Liquidation de la rébellion du pacha de Vidin et ses implications sur la révolte des Serbes ; prétentions du pacha d'Egypte, refusées par le sultan: motivation; refus de la Porte de permettre le passage d'un navire anglais qui transportait des armes pour la Russie, ainsi que d'autres navires commerciaux anglais et russes; motivations ; refus de la Porte de permettre à la France d'acheter des chevaux de chevalerie de l'Empire Ottoman.

\section{Sire,}

Constantinople, 25 Mai 1813

Mon dernier très-humble rapport étoit du 10 de ce mois.

Les dispositions favorables des habitans de Widdin avoient fait bien augurer de la reussite des négociations avec Mollach Pascha, qui, après avoir expliqué ses intentions nullement rebelles, et obtenu sa grace, est sorti avec les siens par une porte de la Ville, pendant que les trouppes du Grand

\footnotetext{
48 Sweden's Diplomatic Mission in Istanbul.

${ }^{49}$ Carl Johan.
} 
Seigneur ${ }^{50}$ entroient par une autre. Il passera par la Capitale pour se retirer dans quelque endroit éloigné de l'Asie. On présume que les Serviens privés de cet appui, suivront bientôt l'exemple de sumission.

L'escadre a defilé jour l'Archipel, précédée par une fregate qui a été expediée à Alexandrie avec un Chambellan du Grand Seigneur, pour porter au Pascha le sabre et la pelisse, l'aigrette de victoire et le titre de Gazi, ou vainqueurs des infidéls. On a aussi parlé du titre de Khan, que le Gouverneur a desirée ; mais que le Grand Seigneur prend lui même, et que n'a été porté par d'autre vassaux que les derniers princes de la Crimée, dependans du Gengis Khan, et heretiers presomptifs de la maison Ottoman.

Un parent des derniers princes de la Georgie, qui est venu ici pour solliciter des secours et un district pour ses adhérens, a été logé chez le Patriarche Grec, et vient d'etre renvoyé avec la Pelise d'honneur, et une manière d'aumone de cinq mille Piastres.

Des batimens anglois arrivé de Malthe et chargé d'armes et de munitions pour les ports Russes de la mer noire, n'ont pu obtenir passage, soit comme contraire à la neutralité, ou à la sureté de l'Empire ${ }^{51}$, si ces armes sont destinées à l'armée que se rassemble sur les frontières. On les disoit d'abord suffisantes pour cent mille hommes, et expediées par le gouvernement anglois (sic !) ; mais il paroit actuellement que cet envoi est une speculation de particuliers, et on ajoute qu'une expedition pareille seroit arrivée dans un port au fond du Golf persiane (sic !), avec des armes pour les Russes à transporter à travers la Perse en guerre avec eux. Je ne sçais quelle inductions on a voulu tirer de ce fait peu vraisemblable.

Le refus de la Porte est consideré comme balancé par un autre au gouvernement francois (sic!), qui auroit demandé de pouvoir acheter des chevaux dans les provinces de l'Empire pour remonter sa Cavalerie. Mais il est accompagné d'autre discussions facheuses, qui semblent embrasser les batimens anglois (sic !) en général, aucun n'ayant passé dans la mer noire depuis la paix, et un entr'autre (sic!), aiant été obligé vendre ici sa cargaison de fruits, gatés pendant l'attente du passage. Un des motifs doit être que le commerce anglois (sic!) ne veut pas se soumettre à la condition de ceder la moitié des cargaisons de retour en bled, au prix fixé par la Porte. Malgré l'acceptation momentanée de cette condition onereuse, des batimens Russes ont été arrêtés aux Dardanelles, comme n'ayant pas delivré la moitié entière. Il est a craindre que la fierté Ottomane se laisse

\footnotetext{
50 Mustafa IV (1807-1808).

51 The Ottoman Empire. 
induire par des suggestions étrangères, dans des difficultés d'une solution toujours plus avantageuse.

Je suis avec le plus profond respect

Sire

de Votre Majesté

le très humble le très fidèle et soumis serviteur et sujet

N. G. Palin.

Sveriges Riksarkivet, Kabinettet/UD Huvudarkivet, E2D, 226, 18111813, Konstantinopel; original in French language.

6

Constantinople, le 25 Juin 1813

Nils Gustaf Palin, [Lars] von Engeström

Sollicite des instructions plus précises, concernant, probablement, la modalité de continuer les démarches diplomatiques pour attirer la Porte dans la coalition anti-française, dans le contexte où la situation militaire avait changé, au mois de mai 1813, en faveur de Napoléon Bonaparte; craintes de la diplomatie suédoise que la Russie, prétextant qu'elle ne collaborait suffisamment avec ses alliés de la coalition anti-française, n'avait pas l'intention d'appuyer la Suède dans la question de la Norvège, possibilité concernant laquelle il fallait informer la Porte ; par conséquent, le diplomate suédois avait adopté une attitude réservée concernant le représentant diplomatique de la Russie à Istanbul.

Constantinople le 25 Juin 1813

Depeche de M[onsieu]r de Palin à S[on] E[cellence] M[onsieu]r le Comte d'Engeström

Les instructions de V[otre] E[xcellence] du 30 Avril et 7 Mai ont en effet rendu ma position un peu difficile par l'embarras de juger de ce qu'il convient de communiquer à la Porte dans le moment présent, que l'état qui semble de nouveau être changé, pourra imposer aux Russes la nécessité d'être humble et loyaux, ou que le resultat des evenemens du mois du Mai ${ }^{52}$ est offusqué par la lenteur du Cabinet de Vienne, pendant que l'Empereur Napoleon proclame l'affermissement de son empire sur l'Allemagne, l'Espagne et l'Italie. J'ai eu même à balancer sur la maniere de communiquer. n'osant pas hasarder l'éclat d'une entrevue personelle avec

\footnotetext{
52 It refers to the victories of the French Army under Napoléon Bonaparte's command in the battles of Lützen and Bautzen against the armies of the anti-French coallition.
} 
le Reis Effendi53 ni rien confier à une Note, ou au vague d'une communication verbale faite par Zeraphino ${ }^{54}$.

Une visite chez le Drogueman ${ }^{55}$ de la Porte à la campagne, quoique masquée par celle à faire à un savant de mes connaissances, qu'il a chez lui, n'ayant pu non plus échaper à l'observation, il ne me restait que la forme d'une instruction par écrit pour Zeraphino. Pour y amener des expressions empruntées des dépeches de V[otre] E[xcellence] je me suis servi d'une Apostille de M[onsieu]r Hegardt ${ }^{56}$ que $\mathrm{j}^{\prime}$ ai fait lire au Drogueman de la Porte, d'après l'habitude de communiquer chaque Courier les lettres intéressantes de ce Chargé d'Affaire qui ont singulierement gagné l'approbation et la confiance.

M[onsieu]r Hegardt fait mention, à la fin de cette apostille, du cri elevé contre les Suédois comme s'ils n'agissent pas assez pour les interets des Alliés, et de ses soupçons que la Cour de S[ain]t Petersbourg à moins d'une necessité, n'avait pas l'intention de remplir ses engagemens envers la Suède par rapport à la Norvège. Ses reflections à ce sujet ne pouvaient que frapper la Porte sans avoir le poids des propres expressions de V[otre] E[xcellence] qu j'ai encore diminuée en m'en servant sans les lui attribuer. J'ai cru pouvoir hasarder une communication en guise de simple éclaircissement de cette apostilee de M[onsieu]r Hegardt et de la maniere que j'ai l'honneur de soumettre très humblement à V[otre] E[xecelence] dans l'apostille très humblement ci-jointe, contenant l'instruction que j'avait donné à Zeraphino. Il devait dire que s'était de son propre mouvement et à mon insçu qu'il fesait lire cette instruction qui ne devait proprement que lui servire de règle pour ses explications verbales. Il a tout executé ainsi, quand le Drogueman de la Porte frapé des expressions de $\mathrm{M}$ [onsieu]r Hegardt en a demandé explication, et ensuite copie de ma piece qui lui a paru d'une importance majeure, mais qu'il n'a pu lire haute au Reis Effendi, absent dans le moment. Je dois rendre compte très humblement de tous ces détails qui auraoint encore la force de ces premieres communications, si par des circonstances, qui je ne puis connaitre encore j'ai eu le malheur de ne pas saisir la juste mesure de ce qu'il convenait de communiquer. J'ai choisi celles des expressions de V[otre] E[xcellence] qui en se pretant le mieux à l'objet apparent d'expliquer celle de $\mathrm{M}$ [onsieu]r de Hegardt met aussi la Porte à même d'apprécier l'état des choses et la valeur des notions qu'elle reçoit ailleurs, ou des mourmures qu'on commence à entendre ici également. En

\footnotetext{
53 Ahmed Effendi (Küçük- Arif).

54 Paul Seraphino.

55 Iacob Arghiropol.

56 Christian Bernhard Hegardt.
} 
terminant le tout par l'allusion à un principe invariable qu'on peut appliquer le changement dans les affaires je me suis aussi assuré des developpement ulterieurs, ou le retour au langage des derniers tems, si j'en obtiens les ordres. En attendant je continuerai de cultiver les missions amis $^{57}$ avec la reserve envers $\mathrm{M}$ [onsieu]r Italinski ${ }^{58}$ qui ma vie retirée facilite.

J'ose solliciter l'indulgence de V[otre] E[xcellence] pou moi aujourdhui, qui ai du consulter les circonstances locales et du moment.

Sveriges Riksarkivet, Kabinettet/UD Huvudarkivet, E2D 226, 18111813, Konstantinopel; copy in French language.

7

Constantinople, le 25 Juin 1813

Nils Gustaf Palin, au [roi de la Suède]

Disputes anglo-turques concernant le problème du commerce anglais sur la Mer Noire; attitude modérée de la diplomatie anglaise et de celle russe concernant la Porte, attitude influencée par le rapport de forces, favorable, alors, à la France, dans la guerre contre la coalition antifrançaise ; craintes de la Porte provoquées par la possibilité de convoquer un congrès des grands pouvoirs européens; attitude ambiguë de la Russie dans le problème de la Norvège; position de l'ambassadeur anglais et du représentant diplomatique ad-interim autrichien d'Istanbul concernant le même problème, ainsi que concernant la question de l'île de Guadeloupe, prétendue par les Suédois.

Constantinople le 25 Juin 1813

\section{Très humble Apostille de Monsieur Palin}

La discussion a été très sérieuse entre l'Ambassadeur d'Angleterre ${ }^{59}$ et la Porte, concernant la Navigation de la Mer-noire (sic !), l'augmentation des droits de Douâne sur differens Articles, et d'autre empiétemens que ce Gouvernement ${ }^{60}$ se permit assés (sic!) arbitrairement. Poussé par les négocians qui jettent des hauts cris, l'Ambassadeur a dû finir par leur faire entendre qu'il ne pouvait pas avancer plus loin sans les Ordres de sa Cour ; puisqu'il ne restait plus qu'à rompre la paix à quoi il n'était pas authorisé.

\footnotetext{
57 The Diplomatic Missions in Istanbul.

58 Andrei Iakovlevici Italinski.

59 Robert Liston; Great Britain's Ambassador at the Ottoman Porte (28.06.1812 - 7.07.1810).

60 High (Sublime) Porte.
} 
L'exemple de $\mathrm{M}[\mathrm{onsieu}] \mathrm{r}$ Arbuthnot ${ }^{61}$ qui avait si légèrement entrainé l'Angleterre dans sa dernière Guerre avec cet Empire62, et la position générale des Affaires, obligent M[onsieu]r Liston a une modération que la Mission Russe observe également dans ce moment, en endurant même de petite humiliations, mais que les Russes seront indubitablement tentés de venger s'ils pourront un jour. Peut-être éspere-t-il même l'appuie de la France pour le rendressement des Griefs après la Paix ${ }^{63}$, car le mecontentimentt est général et très vif dans le consulat Français. Aussi le premier Drogueman Russe m'a-t-il parlé avec une plaisir maligne de l'alarme qu'il a crû appercevoir chez le Reis-Effendi ${ }^{64}$ sur la nouvelle d'un projet de congrés.

Cette Mission ${ }^{65}$ fort polie actuellement, persiste cependant à nier d'avoir jamais reçu aucune information de sa Cour sur ce qui est convenu avec la Suède. Elle garde sa reserve sur a Question de la Norvège. Depuis que M[onsieu]r Liston a été informé par moi du traité conclu avec $l^{\prime}$ Angleterre ${ }^{66}$ il a temoigné sa satisfaction de voir l'indépendance de la Suède affermir par l'aqisition de la Norvège ; et je me suis assuré à la Porte qu'il y tien le même langage. D'autres n'y dissimule peuêtre (sic !) pas l'envie que la prospérité renaissante de la Suède inspire aux Amis, comme

61 Charles Arbuthnot; former ambassador extraordinary and minister plenipotentiary of Great Britain at the Ottoman Porte (3.09.1805- 29/30.01.1807).

62 Continuing to uphold the equilibrium of forces regarding the Eastern Question threatened by France which had the intention of profitting of the outbreak, on December 27, 1806 of the Russian-Turkish War, the ambassador of Great Britain at à Istanbul, Charles Arbuthnot, requested on January 25, 1807 from the Porte on behalf of the British Government to give full satisfaction to Russia as regards the juridical status of the Romanian Principalities and to sever the diplomatic relations with France. The Sultan's refusal was followed by the occupation, a few days later, on January 29, of the Straits of Dardanelles by an English fleet. This led to the war between the Ottoman Empire and the Great Britain which was ended by the end of 1809 with the Peace of Dardanelles, Veniamin Ciobanu, Evoluții politice in Europa Centrală şi de Est (1774-1814) (Iaşi: Editura Junimea, 2007), 191-192.

63 The Russian-Turkush Peace of Bucharest of May 28, 1812.

${ }^{64}$ Ahmed Efendi (Kücük-Arif).

65 Russia's Diplomatic Mission in Istanbul.

${ }^{66} \mathrm{He}$ was referring to the treaty concluded by Sweden with Britain in March 1813 under the terms of which England undertook to support diplomatically or, if necessary, even to annex militarily Norway; at the same time, she agreed to grant a subsidy, worth $£ 1,000,000$ per annum for the duration of the war against France, and to provide the Royal House of Sweden with the possession of the island of Guadeloupe. In return, Sweden commited itself to support the allies in the anti-French coallition with an army of 30,000 soldiers and to accept the establishment of British commercial bases in the ports of Gothenburg, Karlshamn and Stralsund, Dunbar Plunket Barton, Bernadotte (1863-1844), traduit par George Roth (Paris: Payot, 1931), 279-280; Adam Kersten, Historia Szwecji (Wroclaw- Warszawa- KrakówGdansk: Zaklad Narodowy Imienia Ossolinskich Wydawnictwo, 1973), 303. 
aux ennemis ; jusqu'au Ministre d'Autriche ${ }^{67}$ (sic!) m'a dit, quoique sous forme de plaisenterie, que la Suède ne cherche que pêcher en eau trouble, et le dedommagement sur ce que je lui ai appris de nos pretentions sur la Norvège, et que je croyais l'isle de Guadeloupe déjà cédée à la Suède, il m'a repondu plus gravement et du ton d'un Médiateur de la Paix générale : $\mathrm{Qu}$ 'on verra ça à cette Paix.

Je rend compte à S[on] E[xcellence] le Comte d'Engeström ${ }^{6} 8$ de ma dernière communication faite à la Porte, et que Son Drogueman ${ }^{69} \mathrm{~m}^{\prime}$ a fait dire après avoir causé un grand plaisir au Reis Effendi.

Sveriges Riksarkivet, Kabinettet/UD Huvudarkivet, E2D 226, 18111813, Konstantinopel; copy in the French language.

8

Constantinople, le 25 Juin 1813 Nils Gustaf Palin, au roi de la Suède

Echec des négociations anglo-turques dans la question du commerce anglais sur la Mer Noire ; stade du problème serbe ; opérations militaires anglaises dans la Méditerranée Occidentale contre les corsaires.

\section{Sire,}

Constantinople, le 25 Juin 1813

Les dernières instructions de $\mathrm{S}$ [on] $\mathrm{E}$ [xcellence] le Comte $\mathrm{d}^{\prime}$ Engeström ${ }^{70}$ étoient du 27, 30 Avril et 7 Mais, mon dernier très humble rapport etoit du 9 de ce mois.

Le resultat d'une négotietion de l'Ambassade de Angloise, dont ce Courier etoit porteur, n'a pas été statisfasant, la navigation des Anglois sur la mer Noire restant toujours suspendue à cause des conditions, plus onereuse encore que celles qui ont été exigées des Russes, pour la disposition des cargaisons de bled.

Les troupes Ottomanes se mettent en mouvement contre la Servie, sur tois colonnes qui partent de Widin, de la Bosnie et de la Roumelie, la derniere dirigée par Kars Aly Pascha. Un effort aussi enérgique sembleroit devoir ajouter assez de poids à la négociation si longtemps entretenue avec ces Rebelles, pour la terminer biebtôt.

Un firman du Grand Seigneur ${ }^{71}$ sollicité par la France a enjoint au Pascha de Bosnie, de faire restituer à cette puissance la forteresse et le

\footnotetext{
67 Ignaz Lorentz Stürmer.

68 Lars von Engeström.

69 Iacob Arghiropol.

70 Lars von Engeström.
} 
district qui lui ont été enlevées par des Bosniques agissant pour leur compte, et qui dans cette expedition se sont surtout emparé de femmes. Ce genre d'événemens sont comptés ici parmi les bagatelles.

Les operations des Anglois dans l'Archipel contre des corsaires et forbans ne seront gueres autrement considerées, quoiqu'elles ayent entrainé dernierement un borbandement et une descente. Le premier a eu lieu contre isle de Syra, où s'étoit refugiés, avec leur prise, deux corsaires Francois poursuivies par un batiment de guerre Anglois, et où ils ont dressé une batterie avec pavillon Francois. Cette derniere circonstance a motivé le bombardement, qui a detruit le couvent de l'isle et un magasin. Dans l'ardeur singulière des Grecs à s'interesser dans les affaires des puissances etrangères, le parti Russe et Anglois trouve (sic !) aussi qu'on a justement chatié de la sorte les habitans de Syra partisans de la France, et designés comme les Parisiens de l'Archipel. À l'isle de Scopolo, des forbans, sujets Ottomans, pousuivis dans l'interieur du port par les embarcations d'un moindre batiment de guerre Anglois, se sont cachés dernière des rochers et broussailles pour recevoir les anglois (sic !) avec une fusillade qui leur a tué ou blessé une quarantaine d'hommes, sans que les Anglois ayent pu rien faire, ni voir l'ennemi. Le commandant, en restant pour bloquer l'isle, a envoyé avis à une fregatte Angloise stationnée à Smirne, qui est parti pour faire une descente et saisir les coupables. Ils seront sevérement punis par le Capitaine Pascha72, qui se trouvant actuellement dans l'Archipel avec une écadre, prendra sans doute luimême (sic !) dans ses mains la haute police de ces mers du Grand Seigneur.

Le Kaymacan et tout le ministère donnent des fêtes publiques sur les rives du Bosphore au fils du Pascha d'Egypte. Le Grand Seigneur y vient incognito.

La Reine de Sicile n'est pas encore arrivée. Cette princesse qui voyage sous le nom de Comptesse de Castelet a mare, a l'intention de reposer pendant une quinzaine de jours à la campagne de Bujukderé.

$\mathrm{Ci}$ joint une très humble Apostille ${ }^{73}$.

Je suis avec le plus profond respect

Sire

de Votre Majesté

le très humble le très fidèle et soumis serviteur et sujet

\footnotetext{
71 Mahmud II.

72 Gürgi Mehmed Khusrev; capudan pacha (Admiral of the Ottoman Fleet) (April 181121.02.1816).

73 We did not find this piece.
} 
Sveriges Riksarkivet, Kabinettet/UD Huvudasrkivet, E2D, 226, 18111813, Konstantinopel; original in French language.

9

Constantinople, le 10 Juillet 1813

Nils Gustaf Palin, à[Lars von Engeström]

Intérêt manifesté par la Porte concernant l'évolution des rapports suédois - danois dans la question de la Norvège et concernant l'évolution de la guerre entre la France et la Coalition ; intérêt de la Suède concernant la perpétuation des bonnes relations avec la Porte, dans un contexte où elle n'était pas certaine de l'appui des alliés, surtout de la Russie, pour la solution favorable en ce qui la concernait du problème de la Norvège ; reprise des négociations anglo-turques dans la question du commerce anglais dans la Mer Noire.

Constantinople le 10 Juillet 1813

Apostille de M[onsieu]r Palin

$\mathrm{Au}$ départ du dernier Courier le Reis Effendi ${ }^{74} \mathrm{~m}^{\prime}$ a fait donner la

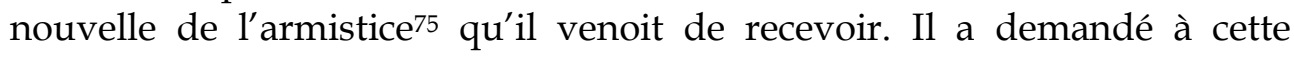
occasion où la Suède en étoit avec le Danemarc, à quoi Séraphino ${ }^{76}$ a repondu qu'il croyoit la discussion devoir aboutir à une guerre. Pour le mettre plus au fait je lui ai communiqué la lettre de M[onsieu]r Hegardt77 du 28 Juin et autres notions reçus par le même Courier. D'après les siennes le Danemarc étoit définitivement lié à la France; il a dit aussi que l'armistice n'améneroit pas la paix ; et c'est le voeu de la porte (sic !) qui dissimule avec peine l'inquiétude qu'elle a conçue de cet armistice. Le refus de S[a] M[ajesté] l'Empereur de Russie ${ }^{78}$ de ratifier la convention du Prince Dolgoruky, le rappel de ce Ministre et l'envoi du Général Suchtelen avec le Baron Weterstedt et les négociateurs Anglois pour porter l'ultimatum des trois Puissances79, d'autre nouvelles et l'espérance sur la Suède qui témoigne toujours la mission Russe, aussi bien que M[onsieu]r de Nesselrode ${ }^{80}$ dans sa dépêche où il lui fait part de l'armistice, toutes ces

\footnotetext{
74 Mehmed Efendi (Kücük-Arif).

75 It probably refers to the armistice concluded by Russia and Prussia with Napoléon Bonaparte, during the summer of 1814 (Anderson, 319).

76 Paul Seraphino.

77 Christian Bernhard Hegardt.

78 Alexander I.

79 Address of Napoléon Bonaparte.

80 Karl Vasilievici Nesselrode.
} 
circonstances paroissent indiquer qu' on cherche à faire justice aux griefs de la Suède ${ }^{81}$. J'ai hésité de donner encore un développement à ma dernière communication faite à la Porte, surtout pendant la cessation (?) et après le changement dans l'état des affaires qui l'a amené. Je suivrai avec exactitude les instructions que S[on] E[xcellence] M[onsieu]r le Comte d'Engeström ${ }^{82}$ m’a donné à ce sujet et ce qui a été déjà dit à la Porte, ni peut qu'avoir un effet salutaire pour affermir la considération de la Suède auprès d'elle.

Le parti Français affecte de repéter que la Suède sera exclue du congrès ${ }^{83}$, apparemment parcequ'elle n'a pa été nommée dans le Moniteur. La depêche de M[onsieu]r de Nesselrode, que le Ministre de Russie ${ }^{84} \mathrm{~m}^{\prime} \mathrm{a}$ fait lire, parlant avec satisfaction de l'entrée de quelques bataillons Suédois à Hambourg, j'ai du faire savoir à M[onsieu]r Italinsky pourquoi cette mesure a été revoquée et après une depêche de Stralsund dont M[onsieu]r Hesgardt m'a fait part et en observant toujours de communiquer à ce Ministre ce que j'apprends par des lettres ostensibles. J'observe aussi ces égards envers l'Ambassadeur d'Angleterre ${ }^{85}$ qui a paru très faché de l'armistice, quoiqu'il le témoigne moins hautement que les autres Anglois, ici. Il m'a dit esperer qu'on avait consulté le prince Royal, avant de la conclure.

Cet Ambassadeur a du reprendre la facheuse négociation sur la navigation de la mer noire (sic !) à l'occasion d'un batiment Anglais, chargé de marchandises pour les ports Russes, qui promettoit assez de profit pour pouvoir supporter la perte sur la cargaison de retour en blé à ceder ici. À la demande des Firmans, il s'est trouvé que la Porte exigeait un péage convenu par aucun Traité. M[onsieu]r Liston aussi bien que l'internonce ${ }^{86}$ ont encore eu a négocier pour le passage de la reine de Sicile, qui est devenu un sujet de discussion d'abord sur les démonstrations d'égard dû à cette Princesse et pour déroger à cette occasion à la défense pour des bâtimens de guerre étrangers de passer les Dradanelles. Comme toute affaire majeure est douteuse, celle çi a été transmise au camp du Grand Vizir ${ }^{87}$, qui l'a renvoyé indécise et à qui il a fallu encore la remettre. Au fond cela ne contrarioit peut être non plus les Ministres d'Angleterre et d'Autriche, le premier étant compris dans la brouillerie entre cette Princesse et sa Cour et l'Autre par une instruction de la sienne.

\footnotetext{
81 In the question of Norway.

82 Lars von Engeström.

83 Future Peace Congress of Vienna.

84 Andrei Iakovlevici Italinski.

85 Robert Liston.

86 Ignaz Lorentz Stürmer.

87 Hurşid Ahmed Pasha.
} 
Sveriges Riksarkivet, Kabinettet/UD Huvudarkivet, E2D 226, 18111813, Konstantinopel; copie en langue française.

\section{References:}

\section{A. Archives}

Sveriges Riksarkivet (Swedish National Archives), Kabinettet (Cabinet) /UD, Huvudarkivet, E2D, 226, 1811-1813, Konstantinopel.

\section{B. Books and articles}

Andersson, Ingvar. A history of Sweden. Translated from the Swedish by Carolyn Hannay. Stockholm: Natur och Kukltur.

Barton, Dunbar Plunket. Bernadotte (1863-1844). Traduit par George Roth. Paris: Payot, 1931.

Ciobanu, Veniamin, Leonidas Rados and Alexandru Istrate, eds. Europe and the Porte. New Documents on the Eastern Question, Volume VII: Swedish Dilomatic Reports 1811-1814. Iaşi: Editura Junimea, 2009.

Ciobanu, Veniamin. Evoluții politice in Europa Centrală şi de Est (1774-1814). Iaşi: Editura Junimea, 2007.

Kersten, Adam. Historia Szwecji. Wroclaw- Warszawa- Kraków-Gdansk: Zaklad Narodowy Imienia Ossolinskich Wydawnictwo, 1973. 